\title{
Taxane and platinum chemotherapy for patients with uterine carcinosarcoma
}

Hiroyuki Yamazaki, Yukiharu Todo, Chisa Shimada, Shinichiro Minobe, Hidenori Kato Division of Gynecologic Oncology, National Hospital Organization, Hokkaido Cancer Center, 4-2 Kikusui, Shiroishi-ku, Sapporo 003-0804, Japan

\section{Objectives:}

To assess the effects of taxane and platinum combined chemotherapy in uterine carcinosarcomas(UCS).

Methods:

We retrospectively examined the records of women with UCS and overall survival(OS)/survival after recurrence(SAR) are demonstrated in Kaplan-Meier method. And disease control rates of chemotherapies were evaluated in women who had measurable disease.

Table. Characteristics.

*Including adjuvant chemotherapies without measurable diseases

\begin{tabular}{|c|c|c|}
\hline & $\begin{array}{c}\text { All eligible } \\
\text { patients }\end{array}$ & $\begin{array}{c}\text { With } \\
\text { measurable } \\
\text { diseases }\end{array}$ \\
\hline $\begin{array}{r}\text { No. of patients } \\
\text { Advanced } \\
\text { Recurrence }\end{array}$ & $\begin{array}{l}45 \\
29 \\
16 \\
\end{array}$ & $\begin{array}{c}28 \\
19 \\
9 \\
\end{array}$ \\
\hline $\begin{array}{c}\text { median } \\
\text { range }\end{array}$ & $\begin{array}{c}64 \\
47-82\end{array}$ & $\begin{array}{c}62 \\
47-77\end{array}$ \\
\hline $\begin{array}{r}\mathrm{IA} \\
\mathrm{IB} \\
\mathrm{II} \\
\mathrm{IIIA} \\
\mathrm{IIIC} 1 \\
\mathrm{IIIC2} \\
\mathrm{IVB} \\
\end{array}$ & $\begin{array}{c}9 \\
3 \\
4 \\
2 \\
4 \\
4 \\
19 \\
\end{array}$ & $\begin{array}{c}4 \\
2 \\
3 \\
0 \\
1 \\
2 \\
16 \\
\end{array}$ \\
\hline $\begin{array}{r}\text { Surgery } \\
\text { Biopsy only } \\
\text { Histerectomy } \\
+ \text { LND/LN biopsy } \\
\end{array}$ & $\begin{array}{c}3 \\
18 \\
24 \\
\end{array}$ & $\begin{array}{c}3 \\
13 \\
12 \\
\end{array}$ \\
\hline $\begin{array}{l}\text { Nx } \\
\text { No } \\
\text { N1 }\end{array}$ & $\begin{array}{c}21 \\
15 \\
9 \\
\end{array}$ & $\begin{array}{c}16 \\
9 \\
3 \\
\end{array}$ \\
\hline $\begin{array}{l}\text { Previous } \\
\text { Taxan+Platinum* } \\
\text { Yes } \\
\text { No }\end{array}$ & $\begin{array}{l}25 \\
20\end{array}$ & $\begin{array}{c}21 \\
7\end{array}$ \\
\hline
\end{tabular}

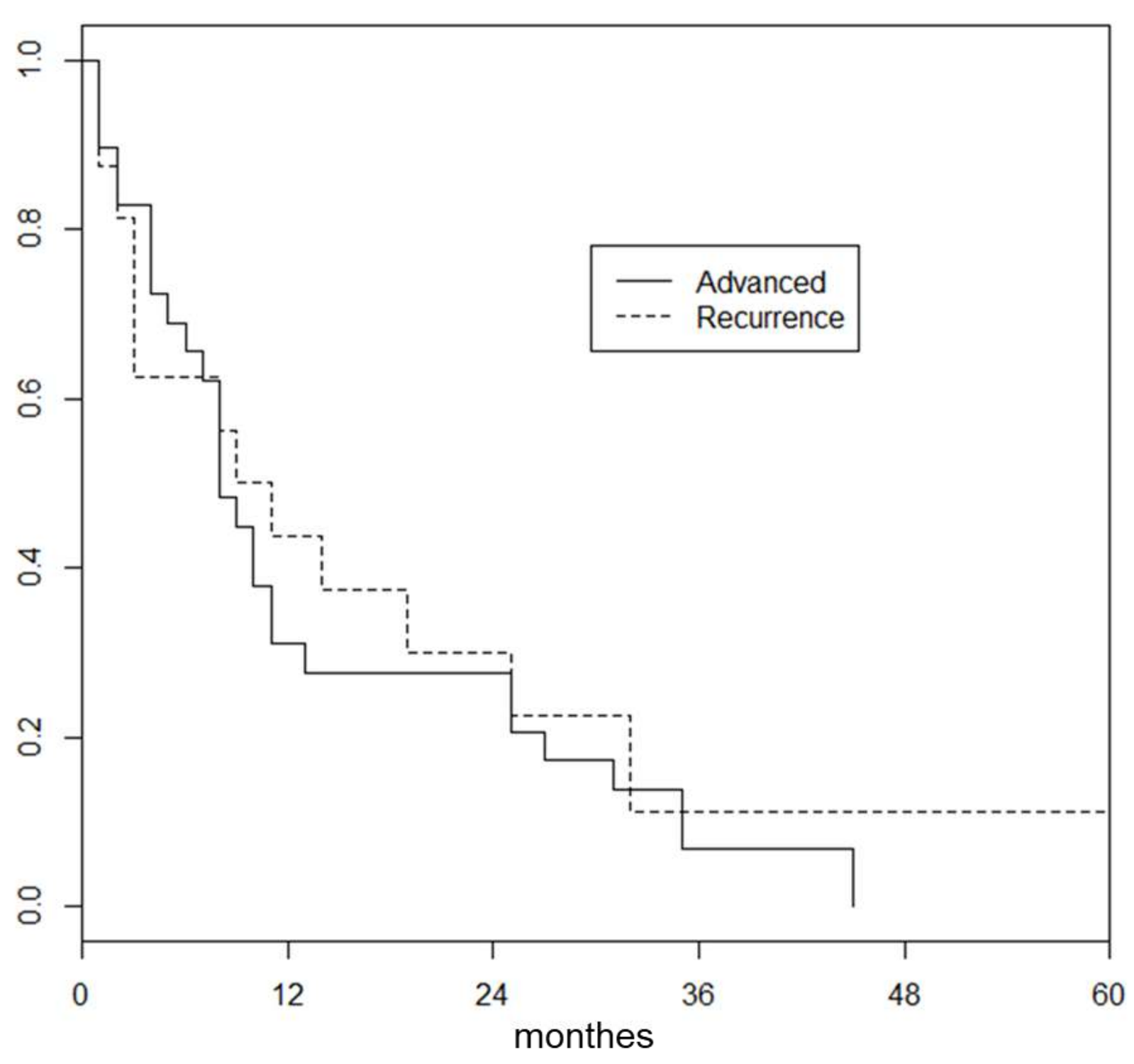

Figure. Kaplan-Meier survival curves.

:OS in advanced group, SAR in recurrence group.
Results:

45 women were eligible among seventy-five patients with UCS in 1987-2016. 29 had advanced stage (III or IV) and 16 had recurrence disease. Their overall survival and survival after recurrence was 8 months (median, range 1-45) and 10 months (median, range 1-68). The two-year survivals were $20 \%$ and $22 \%$, respectively. 28 patients had measurable disease when treated with chemotherapy, which accounted for 39 regimens. The disease control rate of taxane and platinum combined chemotherapy was $38.9 \%$.

Conclusions:

Taxane and platinum combined chemotherapy for advanced or recurrent UCS showed less activity than previously reported although this is a single center retrospective study in the small number.

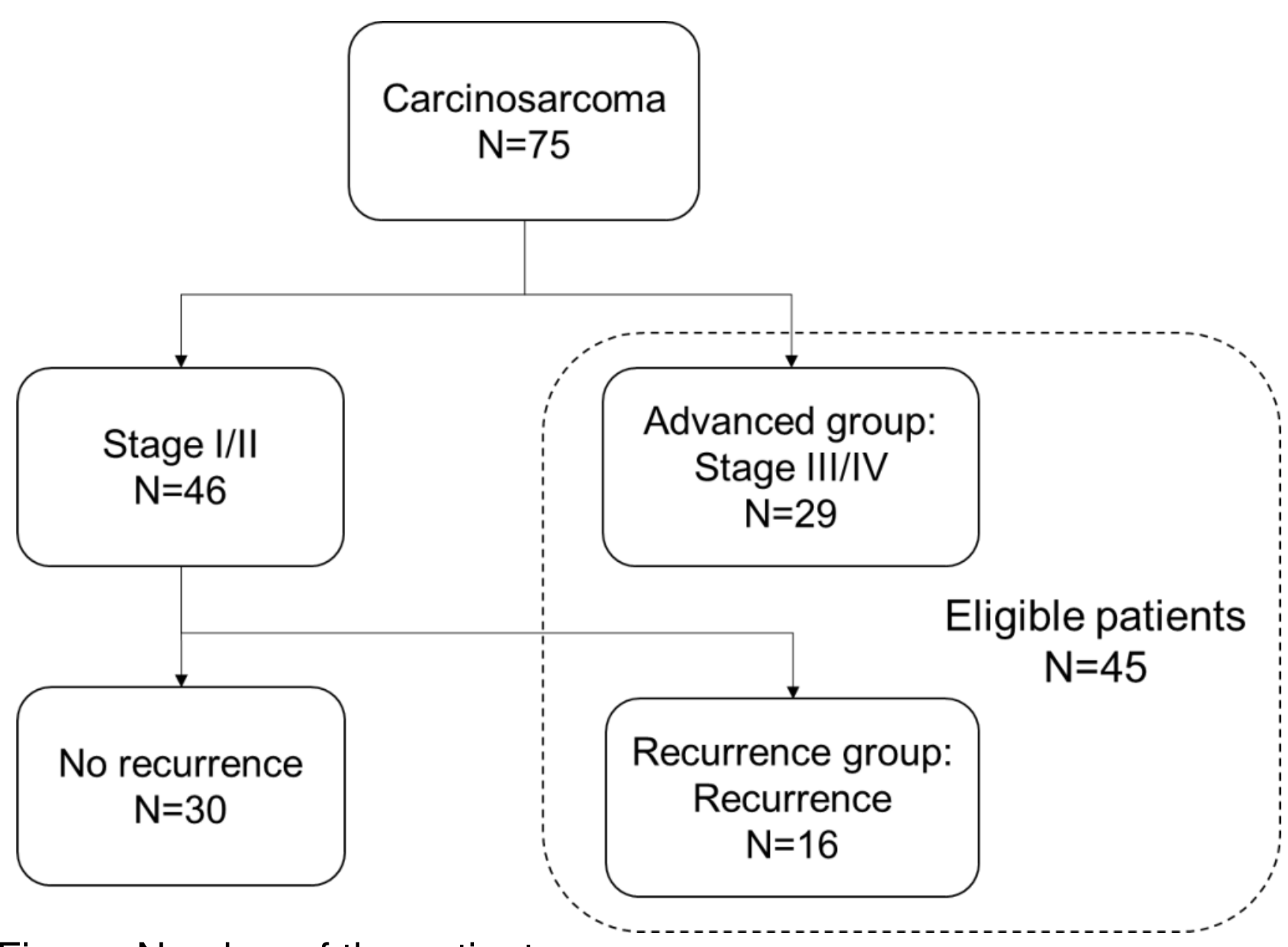

Figure. Number of the patients.

Table. Response to the chemotherapies grouped by the key-drugs

\begin{tabular}{|c|c|c|c|c|c|c|}
\hline & & CR & PR & SD & \multirow{2}{*}{ PD } & \multirow{2}{*}{$\begin{array}{c}\text { Previous } \\
\text { chemotherapy }\end{array}$} \\
\hline & & \multicolumn{3}{|c|}{$\begin{array}{c}C R+P R+S D \\
\text { (Disease control rate) }\end{array}$} & & \\
\hline \multirow{2}{*}{$\begin{array}{l}\text { Taxane and } \\
\text { platinum }\end{array}$} & \multirow{2}{*}{18} & 2 & 2 & 3 & \multirow{2}{*}{11} & \multirow{2}{*}{$3(16.7 \%)$} \\
\hline & & & 38.9 & & & \\
\hline \multirow{2}{*}{ Gemcitabine } & \multirow{2}{*}{8} & & 1 & & \multirow{2}{*}{7} & \multirow{2}{*}{$8(100 \%)$} \\
\hline & & \multicolumn{3}{|c|}{$1(12.5 \%)$} & & \\
\hline \multirow{2}{*}{ Adriamycin } & \multirow{2}{*}{6} & & & 1 & \multirow{2}{*}{5} & \multirow{2}{*}{$4(66.7 \%)$} \\
\hline & & & 16.7 & & & \\
\hline \multirow{2}{*}{$\begin{array}{c}\text { Platinum } \\
\text { (without taxane) }\end{array}$} & \multirow{2}{*}{4} & & 1 & & \multirow{2}{*}{3} & \multirow{2}{*}{$2(50 \%)$} \\
\hline & & \multicolumn{3}{|c|}{$1(25 \%)$} & & \\
\hline \multirow{2}{*}{ Pazopanib } & \multirow{2}{*}{3} & & 1 & 1 & \multirow{2}{*}{1} & \multirow{2}{*}{$2(66.7 \%)$} \\
\hline & & \multicolumn{3}{|c|}{$2(66.7 \%)$} & & \\
\hline
\end{tabular}

